\title{
Wavelet based multicarrier CDMA system
}

\author{
Nasser Hamad ${ }^{1}$, Maen Takruri², Mahdi Barhoush ${ }^{3}$ \\ ${ }^{1,2}$ Department of Electrical, Electronics and Communication Engineering, American University of Ras Al Khaimah, UAE \\ ${ }^{3}$ Department of Telecommunication Engineering, Arab American University-Jenin, Palestine
}

\begin{tabular}{l}
\hline \hline Article Info \\
\hline Article history: \\
Received Jun 27, 2018 \\
Revised Mar 3, 2019 \\
Accepted Mar 21, 2019
\end{tabular}

Keywords:

Discrete fourier transform Discrete wavelet transform Multicarrier modulation Peak to average power

\begin{abstract}
Emerging demands for high data rate services, high user capacity and low power consumption systems are the key driving forces behind the continued technology evolution in wireless communications. Multicarrier Modulation techniques support variety of services requiring different data rates and different QoS (quality of service) levels. Multicarrier CDMA is a wireless communication system that can be seen as a combination of direct sequence CDMA and Orthogonal Frequency Division Multiplexing techniques. The main benefits of this system are its robustness to inter symbol interference and multipath propagation in fading channels. This paper studies and simulates the Discrete Wavelet Transform based Multicarrier CDMA and compares it with the Discrete Fourier Transform based one using different number of sub carriers, and different modulation techniques. The results shows that the Wavelet based system outperforms the Fourier based one since it has lower bit error rate $B E R$ performance, lower peak to verage power ratio $P A P R$ and higher user capacity.
\end{abstract}

Copyright () 2019 Institute of Advanced Engineering and Science. All rights reserved.

\section{Corresponding Author:}

Nasser Hamad,

Department of Electrical, Electronics, and Communication Engineering,

American University of Ras Al Khaimah (AURAK),

Al Breirat, Ras Al Khaimah, United Arab Emirates.

Email: Nasser.hamad@aurak.ac.ae

\section{INTRODUCTION}

The demand on higher data rate wireless communication services is expected to increase rapidly. Therefore, researchers all over the world are motivated to define new generations of wireless systems that have better data rates and quality of service than existing ones. One major goal of the new generation systems is to integrate the existing standards into a common platform that can be achieved through an adoption of flexible air interface with future proof and can open the door for new technologies. Due to its high spectral efficiency, Multicarrier CDMA (MC-CDMA) has become an option for high data rate transmission systems in high mobility [1-3]. MC-CDMA is a wireless communication system that can be seen as a combination of direct sequence CDMA and Orthogonal Frequency Division Multiplexing techniques.

Aiming at increasing data rates of wireless communication with high performance, OFDM technology is implemented by either Inverse Fast Fourier Transform (IFFT) or Discrete Wavelet Transform (DWT) [4-6]. IEEE standardization group adopted OFDM as the basis for their new 5-GHz standard, targeting a range of data stream from 6 up to 54 Mbps in 1998 [7]. Conventional OFDM system implemented by IFFT at the transmitter and FFT at the receiver was adopted to increase data rates of wireless communication with higher performance. However, FFT has a major drawback arising from using rectangular window which creates sidelobes, as the pulse shaping function used to modulate each subcarrier extends to infinity in the frequency domain which reduces the spectrum efficiency. Intercarrier interference (ICI) and intersymbol interference (ISI) can be avoided by inserting a Cyclic Prefix (CP) to the head of OFDM symbol such that the delay spread of the channel becomes longer than the channel impulse response which brings the disadvantage of further reducing the spectral efficiency [8]. 
Finally, high peak to average power ratio (PAPR) that causes nonlinear distortion in the transmitted OFDM signal is also considered a major disadvantage associated with OFDM [9]. Due to the aforementioned drawbacks, an alternative method is to use the wavelet transform to replace the IFFT and FFT blocks [10]. Discrete Wavelet Transform (DWT) is a mathematical transform that provides a time-frequency representation of a discrete time domain signal using special signals called wavelets having specific properties that make them useful for signal processing. Moreover, DWT have proven to be successful for the detection and estimation of signals. Since in FFT-OFDM systems, signals only overlap in the frequency domain while DWT-OFDM signals overlap both in time and frequency domains, so there is no need for the $\mathrm{CP}$ as in the FFT-OFDM case. In contrast to FFT, which uses a single analysis window, the Wavelet transform uses short windows at high frequencies and long windows at low frequencies. That's why the wavelet transform is implemented using Low Pass Filter (LPF) and High Pass Filter (HPF), perfect reconstruction of received signals is acheived. In literature, these two filters are also called subband coding since the signals are divided into sub-signals of low and high frequencies. A wavelet approach, moreover, is implemented for the reduction of PAPR and computational complexity [11].

In the last decade, several research works have been made on the comparisons between DWTOFDM and FFT-OFDM systems [12], there results show performance enhancement of DWT-OFDM over FFT-OFDM. In order to enhance the performance of OFDM technique based on Discrete Wavelet Transform DWT-OFDM, Forward Error Correction (FEC) code and Viterbi decoder of high speed decoding capabilities was introduced in [4]. Others have made comparisons between several mother functions of wavelet [13]. The work in [14] studied the BER performance of OFDM discrete wavelet transform, although they compared the performance for both channels AWGN and dispersive channels, they did not show the performance enhancement for different combinations of employed modulation techniques and the required data rates. Finally, the work in [15], compared the results of DWT based MC-CDMA with the results of DFT based MC-CDMA. The simulation results in [15] showed that DWT based MC-CDMA system has better performance than DFT based MC-CDMA at the cost of higher computational complexity. The performance measurement in [15] was carried out for different vehicles or user speeds.

Pratima, et.al, in [16], compare the PAPR using SLM with Cyclic \& Linear block codes. However, they do not consider the case of comparing PAPR for the DFT based MC-CDMA and DWT based MCCDMA. Moreover, the results in [17] show the distribution of the PAPR for every decomposition level to obtain the best decomposition level for each wavelet family, and their simulation results are given for PAPR distribution of a DWT-OFDM system using two clipping nonlinearity functions and compare them with DWT-OFDM and DFT-OFDM systems. Unlike our comprehensive work, that shows results of the PAPR for several modulation techniques for both DFT- and DWT- based MC-CDMA system.

In this paper, the main objective is to evaluate the through simulation the performance of the integration of DWT with multicarrier system as a replacement transform for DFT in a MC-CDMA system. The performance of the DWT based MC-CDMA system is compared with the DFT based MCCDMA in terms of Bit Error Rate ( $B E R)$ and Peak to Average Power Ratio (PARP).

This paper is organized as follows: In Section 2, we discuss the FFT-OFDM. DWT-OFDM system is presented in Section 3. In Section 4, Multicarrier modulation techniques are introduced. Simulation results are presented and discussed in Section 5, and we conclude this work in Section 6.

\section{FFT BASED OFDM SYSTEM}

Using of Discrete Fourier Transform (DFT) to parallel data transmission as part of the modulation and demodulation process eliminates the need for banks of sub channel oscillators and coherent demodulators and so reduces the system complexity. The DFT based technique was called orthogonal frequency division multiplexing OFDM. OFDM has been exploited for wideband data communications, Asymmetric Digital Subscriber Lines (ADSL), and HDTV terrestrial broadcasting.

Let $R_{b}$ be the transmitted data rate and $N_{c}$ be the number of OFDM sub channels, then the system uses $N_{c}$ data bits to modulate each of the system sub channels and sends them simultaneously over lower symbol rate $R_{s}$, hence each bit now can be sent in longer symbol duration $T_{\mathrm{s}}=N_{c} / R_{b}$. The spectral spacing $\Delta f$ between carriers should be equal to $1 / T_{s}$ resulting in a minimum bandwidth $N_{c}$. Moreover, assume $d_{k}$ is the $k^{\text {th }}$ bit of the parallel data stream used to modulate the corresponding $k^{\text {th }}$ subcarriers at frequency $f_{k}=f_{c}+k \Delta f$, then the OFDM complex signal $m(t)$ is the sum of all sub channels signals given by

$$
m(t)=\sum_{k=0}^{N_{c}-1} d_{k} e^{j 2 \pi\left(f_{c}+k \Delta f\right) t}
$$


After sampling of the signal $m(t)$ at $1 / T_{s}$ sampling rate, expression in (1) is rewritten as

$$
m\left(n T_{s}\right)=\sum_{k=0}^{\mathrm{N}_{\mathrm{c}}} d_{k} e^{j 2 \pi\left(f_{c}+k \Delta f\right) \mathrm{nT}_{\mathrm{s}} / N_{c}}
$$

Let $f_{c}=0$, and recall that $\Delta f T_{s}=1$, then

$$
m\left(n T_{s}\right)=\sum_{k=0}^{N_{c}-1} d_{k} e^{j 2 \pi k n \mathrm{~T}_{\mathrm{s}} / \mathrm{N}_{\mathrm{c}}}
$$

Inverse (DFT) IDFT of the $N_{c}$ parallel OFDM data block is described in (3) and implemented by Inverse Fast Fourier Transform (IFFT) which is faster and less complex method. Figure 1 shows the block diagram of the OFDM system.

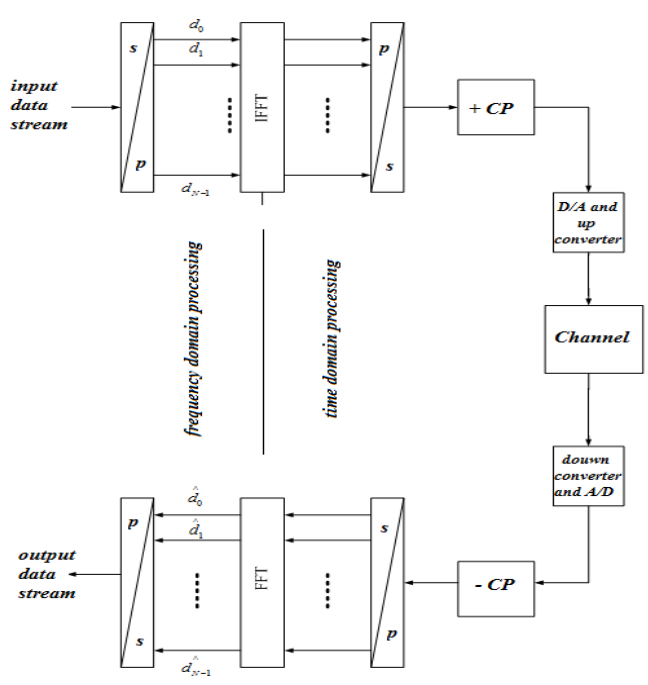

Figure 1. OFDMA system block diagram

At the transmitter side, the serial input data stream is converted into parallel data, $N_{c}$ bits at a time. These $N_{c}$ bits are defined in the frequency domain such that their amplitudes and phases are those of the sub channels of the OFDM system. IFFT then transforms this spectrum to its corresponding time domain signal, the parallel data is converted to serial data and cyclic prefix extinction is added to eliminate ISI. The signal is then converted from digital to analogue using low pass filter and up converted to OFDM frequency band. In the receiver side, after the transmitted signal is convolved with the channel impulse response, it is down converted and digitized, then the cyclic extension is removed and FFT operation is performed on the received parallel $N_{c}$ time domain samples to recover the transmitted frequency samples using appropriate decision circuit. It is worthy to mention that the total transmitted block duration $T$ is equal to the original symbol duration $T_{s}$ plus the inserted cyclic prefix interval $T_{g}$, i.e., $T=T_{s}+T_{g}$.

As a result, we can see that OFDM can be implemented efficiently using IFFT/FFT which perform the orthogonallity between the sub channels and cancel the necessity of tuned sub channels receiver filters used for conventional FDM systems. Moreover, the high spectral efficiency of OFDM compared to conventional modulation scheme is another attractive feature. The drawback of such implementation is the less bandwidth efficiency due to the use of cyclic prefix and the less power efficiency due to $P A R P$.

\section{DWT BASED OFDM SYSTEM}

The IFFT/FFT and cyclic prefix blocks at the OFDM block diagram shown in Figure 1 could be replaced with IDWT/DWT (Inverse Discrete Wavelet Transform) to form the wavelet based OFDM system. The Continous Wavelet Transform (CWT) is defined as

$$
F_{W T}(\tau, s)=\int_{-\infty}^{\infty} f(t) \frac{1}{\sqrt{s}} \psi^{*}\left(\frac{t-\tau}{s}\right) d t
$$


where $f(t)$ is the time-domain signal to be transformed, $s$ is the scale, $\tau$ is the translation, $\psi$ is the mother wavelet and $F$ is the continuous wavelet transform. There are different sets of mother wavelets with different properties that make them suitable for different applications. The time-frequency representation of this transform appears in scale and translation, the translation $\tau$ contains the time information while the scale $s$ contains the spectrum information which indicates the reciprocal of frequency $(1 / f)$. In terms of frequency, low frequencies (high scales) correspond to a global information of a signal, whereas high frequencies (low scales) correspond to a detailed information of a hidden pattern in the signal.

A time-scale representation of a digital signal can be obtained using digital filtering techniques, decomposition coefficient are computed with a fast algorithm that cascades discrete convolution with $h$ and $\mathrm{g}$, and subsamples the output. In DWT, iterative half band analysis lowpass and highpass filters, $h$ and $\mathrm{g}$ respectively, are used to compute the approximation $a$ and detail information $d$ for the discrete signal $x[n]$ in every level of decomposition as shown in Figure 2.
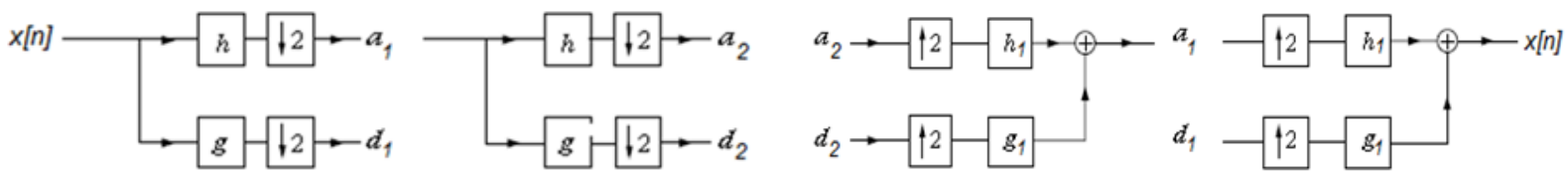

Figure 2. Two levels discrete wavelet decomposition, transmitter and reciver sides

The output of each filter is a discreet signal that contains only half of the frequency band, this doubles the frequency resolution since the uncertainty in frequency is reduced by half. Relying to Nyquist's rule, no loss of information occurs if half of the samples are discarded from each output since they are redundant information, sub-sampling by 2 , halves the time resolution as the entire signal is now represented by only half the number of samples. Hence, precise time resolution at high frequencies, and precise frequency resolution at low frequencies. Expressions in (5) and (6), shows the decomposition process.

$$
\begin{aligned}
& a_{2}[p]=\sum_{n=-\infty}^{\infty} \mathrm{h}[n-2 p] a_{1}[n] \\
& d_{2}[p]=\sum_{n=-\infty}^{\infty} \mathrm{g}[n-2 p] a_{1}[n]
\end{aligned}
$$

The above procedure which is known as Mallat algorithm [18] can be repeated for the desired level of decomposition that depends on the length of discrete signal $x[n]$. The DWT of the original signal is then obtained by concatenating all the coefficients, $a$ and $d$, starting from the last level of decomposition.

Figure 2 shows the inverse discrete wavelet transform IDWT that reconstruct the original signal $x[n]$ from the wavelet coefficients. The approximation and detail coefficients at every level are up-sampled by two, passed through the lowpass and highpass synthesis filters, $h_{1}$ and $g_{1}$ respectively, and then added as shown

$$
a_{1}[p]=\sum_{n=-\infty}^{\infty} \mathrm{h}_{1}[p-2 n] a_{2}[n]+\sum_{n=-\infty}^{\infty} \mathrm{g}_{1}[p-2 n] d_{2}[n]
$$

\section{MULTICARRIER SYSTEM}

A multiple access technique that combines both multicarrier and CDMA is called a MC-CDMA in which user are allowed to share the same bandwidth at the same time and using signature codes to separate each user specific data. The high rate data symbols are serial to parallel converted to get a parallel low rate sub streams, then the signature codes are used to spread the data symbols for each sub stream into its corresponding OFDM sub channel. Moreover, MC-CDMA is a serial concatenation of DS-CDMA and OFDM systems, the OFDM system could be based on Fourier transform or Wavelet transform to modulate its sub channels to end with a Wavelet or Fourier based MC-CDMA as shown in Figure 3. 


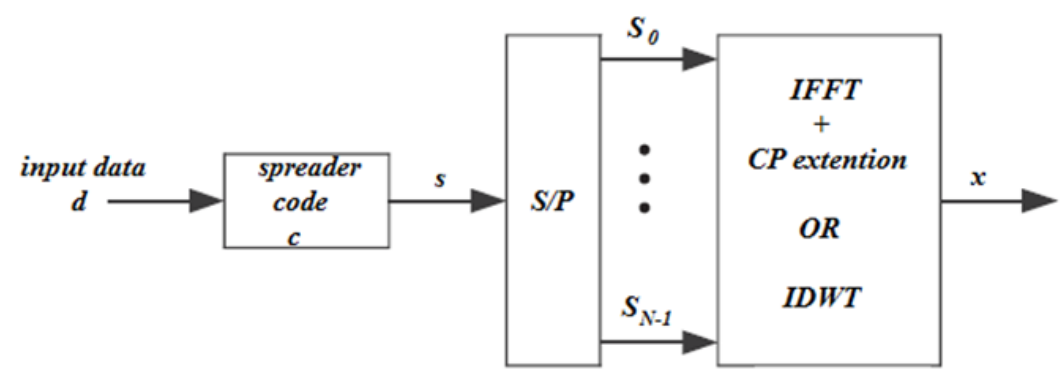

Figure 3. Wavelet or Fourier based MC-CDMA system

\subsection{MC-CDMA system model}

The transmitter part of MC-CDMA for a user in the uplink transmission or one active user in the downlink transmission is shown. For $K$ active users, $d^{k}$ and $c^{k}$ denote the single complex valued data symbol and the specific signature code assigned to the user $k$, respectively, where $c^{k}=\left\langle c_{0}^{k}, c_{1}^{k}, \ldots, c_{G-1}^{k}\right\rangle$ and $G$ is the spreading factor (Sometimes it is referred to as processing gain which is the ratio between the bit duration and the chip duration ).

If the rate of the serial data symbols $d^{k}$ is $R_{d}=1 / T_{d}$, then the rate of the serial spread data $s^{k}$ is $G R_{d}$ where $s^{k}$ is given by

$$
s^{k}=d^{k} c^{k}=\sum_{i=0}^{G-1} d^{k} c_{i}^{k}=\left\langle S_{0}^{k}, S_{1}^{k}, \ldots, S_{G-1}^{k}\right\rangle .
$$

The spread data symbol are serial to parallel converted to modulate $G$ sub carriers of the OFDM system using either IFFT or IDWT, i.e. each data symbol is spread over $G$ subcarriers. In the case where the processing gain $G$ equals the number of the OFDM subcarriers $N_{c}$, one data symbol per user is transmitted in one OFDM symbol.

In the receiver side, the inverse OFDM of the received signal which is the transmitted one from all active users $K$ convolved with channel impulse response $h(t)$ in addition to additive white Gaussian noise AWGN is performed. Provided that the number of OFDM subcarriers is large and the guard interval is long enough to absorbs all the echoes (no ISI), the channel response of each subcarrier can be modeled as a flat fading channel and the received signal after the OFDM system is given by

$$
r=\sum_{k=0}^{K-1} H^{k} s^{k}+n=\left\langle R_{0}, R_{1}, \ldots, R_{N_{c}-1}\right\rangle
$$

where $H^{k}$ represents the coefficients of the sub channels for each user $k, s^{k}$ is obtained from (8) and $n$ is the noise vector of length $N_{c}$. The received signal in the MC-CDMA can be written as

$$
r=H s+n=\left\langle R_{0}, R_{1}, \ldots, R_{N_{c}-1}\right\rangle
$$

where $H$ represents $N_{c} \times N_{c}$ channel matrix. The received vector $r$ that is fed to a data detector to estimate the transmitted data can be written as,

$$
r=\sum_{k=0}^{K-1} \sum_{i=0}^{N_{c}-1} d^{k} c_{i}^{k} H_{i}^{k}+\sum_{i=0}^{N_{c}-1} n_{i}
$$

In single user detection strategy, the received vector $r$ is compensated using simple multiplier equalization with frequency domain gain factor $Q_{i}$ for each sub carrier, and then despread using code $c^{k^{\prime}}$ of the user of interest $k^{\prime}$ to get the noisy data symbol ${d^{\prime}}^{\prime}$ as

$$
\begin{aligned}
d^{\prime k^{\prime}} & =\sum_{k=0}^{K-1} \sum_{i=0}^{N_{c}-1} d^{k} c_{i}^{k} c_{i}^{k^{\prime}} H_{i}^{k} Q_{i}^{k}+\sum_{i=0}^{N_{c}-1} c_{i}^{k^{\prime}} n_{i} Q_{i} \\
& =d^{k^{\prime}} \sum_{i=0}^{N_{c}-1} H_{i}^{k} Q_{i}^{k}+\sum_{\substack{k=0 \\
k \neq k^{\prime}}}^{K-1} d^{k} \sum_{i=0}^{N_{c}-1} c_{i}^{k} c_{i}^{k^{\prime}} H_{i}^{k} Q_{i}^{k}+\sum_{i=0}^{N_{c}-1} c_{i}^{k^{\prime}} n_{i} Q_{i} .
\end{aligned}
$$

The first term represents the desired signal component, the second term is the multiple access interference (MAI), and the last one is the noise component. The performance of the system can be improved by the multiuser detection technique that uses all the available information at the receiver, such as the 
spreading codes of all the users and their channel impulse response. The most known multiuser detection is maximum likelihood detection and interference cancelation that detects and subtracts the interfering signals from the received signal before finding the signal of interest, for more details on multiuser detection strategies and cancellation techniques $[19,20]$.

\subsection{Peak to average power ratio}

The variation of the envelope of the time domain OFDM signal can be defined by the peak to average power ratio $P A R P$ given by

$$
P A R P=\max \left\{\left|m_{i}\right|^{2}\right\} /\left[\frac{1}{N_{c}} \sum_{i=0}^{N_{c}-1}\left|m_{i}\right|^{2}\right],
$$

where $m_{\mathrm{i}}, i=0,1, \ldots, N_{c}-1$ are the time domain samples of the OFDM signal computed by the inverse discrete Fourier transform IDFT or the inverse discrete wavelet transform IDWT. High PAPR is actually a nontrivial issue for OFDM systems because it increases the complexity of the analog to digital and digital to analog converters and reduces the efficiency of the power amplifier, i.e. when the time domain signal traverses from a low instantaneous power waveform to a high power waveform, it results in a high out of band distortion power, unless the transmitter's power amplifier exhibits an extremely high linearity which is difficult to achieve, and so several techniques are used to reduce the PARP or improving the amplification stage of the amplifier.

\section{SIMULATION RESULTS}

Our main goal of this research is to give a full description of wavelet based MC-CDMA and to compare the performance with the Fourier based MC-CDMA in both downlink and uplink transmission mode. The system simulation represents the synchronous downlink and uplink transmission, both the wavelet and Fourier based MC-CDMA systems used the orthogonal Walsh codes generated using Hadamard transform to spread the users data. The modulation techniques used are M-QAM and M-PSK and we applied the single user detection technique. The channel is modeled as Additive White Gaussian Noise AWGN Channel. Table 1 shows our simulation parameters.

\begin{tabular}{cc} 
Table 1. MC-CDMA system simulation parameters \\
\hline Parameter & Value \\
\hline Data length & 180 Kbit \\
Number of channels & $128,256,512,1024$ \\
Number of users & variable \\
Modulation techniques & M-QAM, M-PSK \\
Detection strategy & Single user detection \\
Signature codes & Orthogonal codes (Walsh) \\
Transmission & Downlink and Uplink \\
\hline
\end{tabular}

In this simulation, we compare the performance of the wavelet based MC-CDMA over the Fourier based one in terms of BER and PAPR using different types of wavelet families, modulation techniques, variable number of users and different number of carriers as given in Table 1.

Figure 4 shows the wavelet based system (Haar) performs better than the Fourier based one with different M-PSK modulation techniques over the AWGN channel. Figure 5 shows that the wavelet system is better than the Fourier transform system with different M-QAM modulation techniques in both uplink and downlink transmission.

Different wavelet families such as Daubechies, Symlets, Biorthogonal, reverse Biorthogonal and Coiflets are employed in discrete wavelet transform and their performances are with discrete Fourier transform as shown in Figure 6 where 400 active users in 512-channel system are simulated. Moreover, Figure 6 shows the superiority of wavelet transform over the Fourier one, it also shows that 'Haar' has the best performance in the wavelet families. 


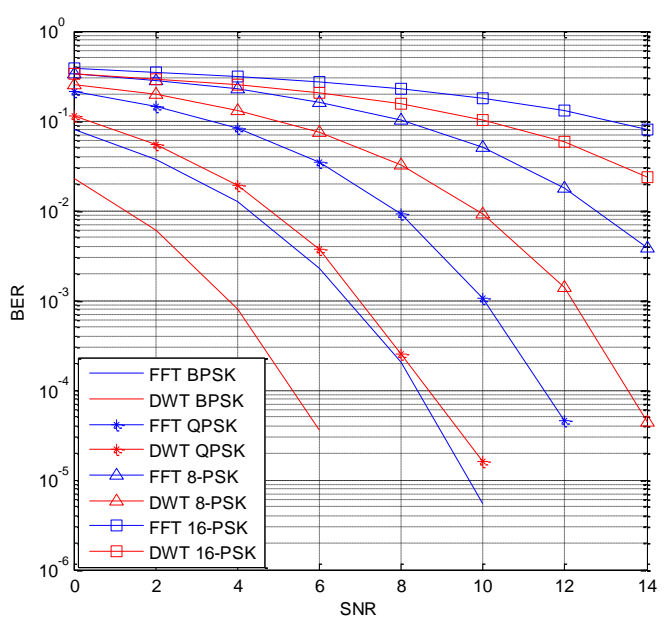

Figure 4. BER performance with different M-ary modulation techniques
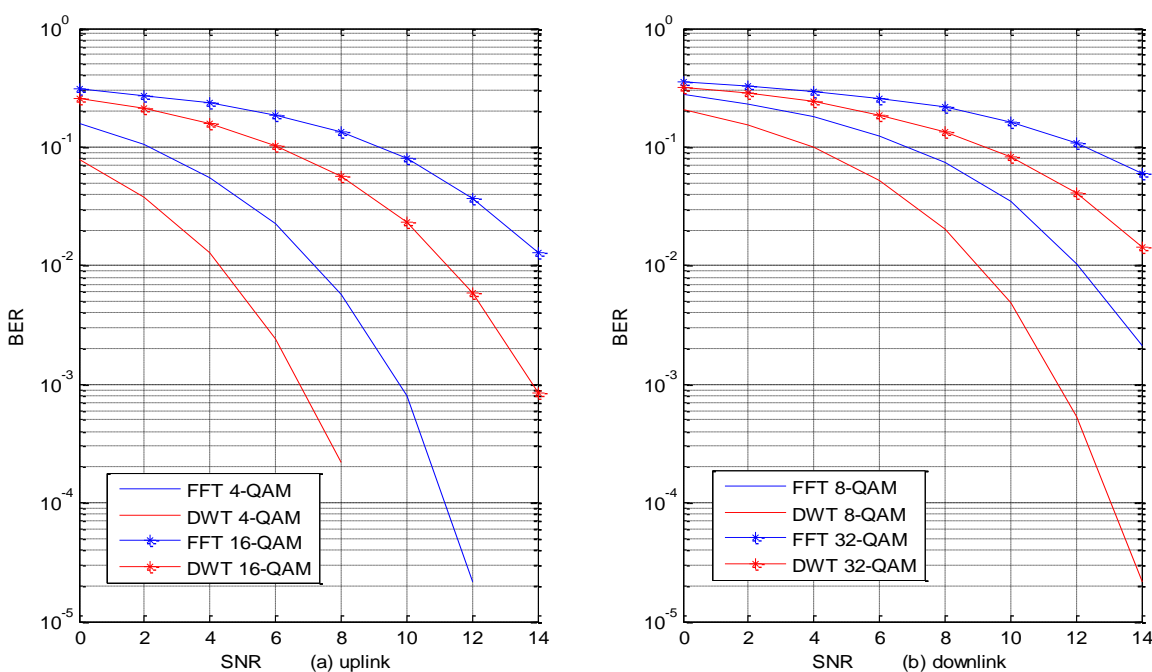

Figure 5. BER performance with different M-QAM modulation technique

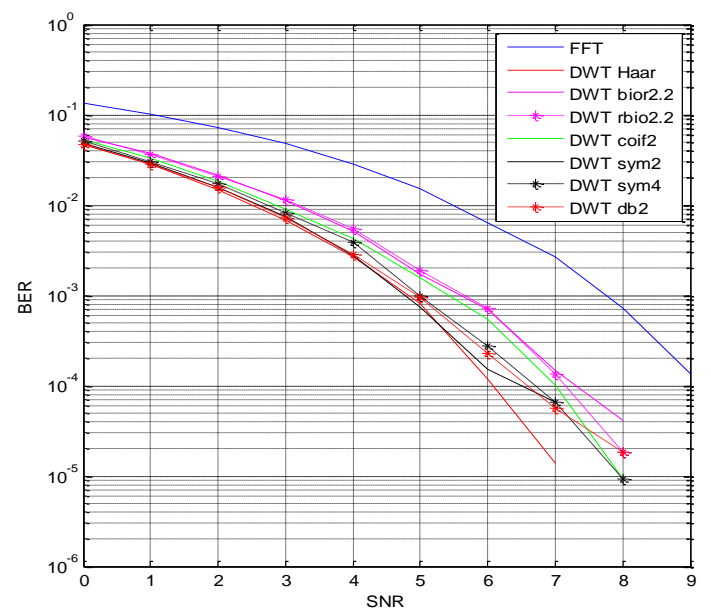

Figure 6. BER performance for different wavelet families 
Figure 7 shows the PAPR for both the wavelet and Fourier based MC-CDMA systems in the downlink and uplink transmission at different number of sub carriers and with different modulation techniques. From Figure 7, one can see that the PAPR is independent of the modulation techniques in both cases. Moreover, the $P A P R$ for both Wavelet and Fourier is slightly propotional to the number of sub carriers in the downlink transmission whereas, in the uplink transmission, the PAPR of the wavelet system is constant with the number of sub carriers and is extremely propotional to the number of sub carriers for the Fourier case.
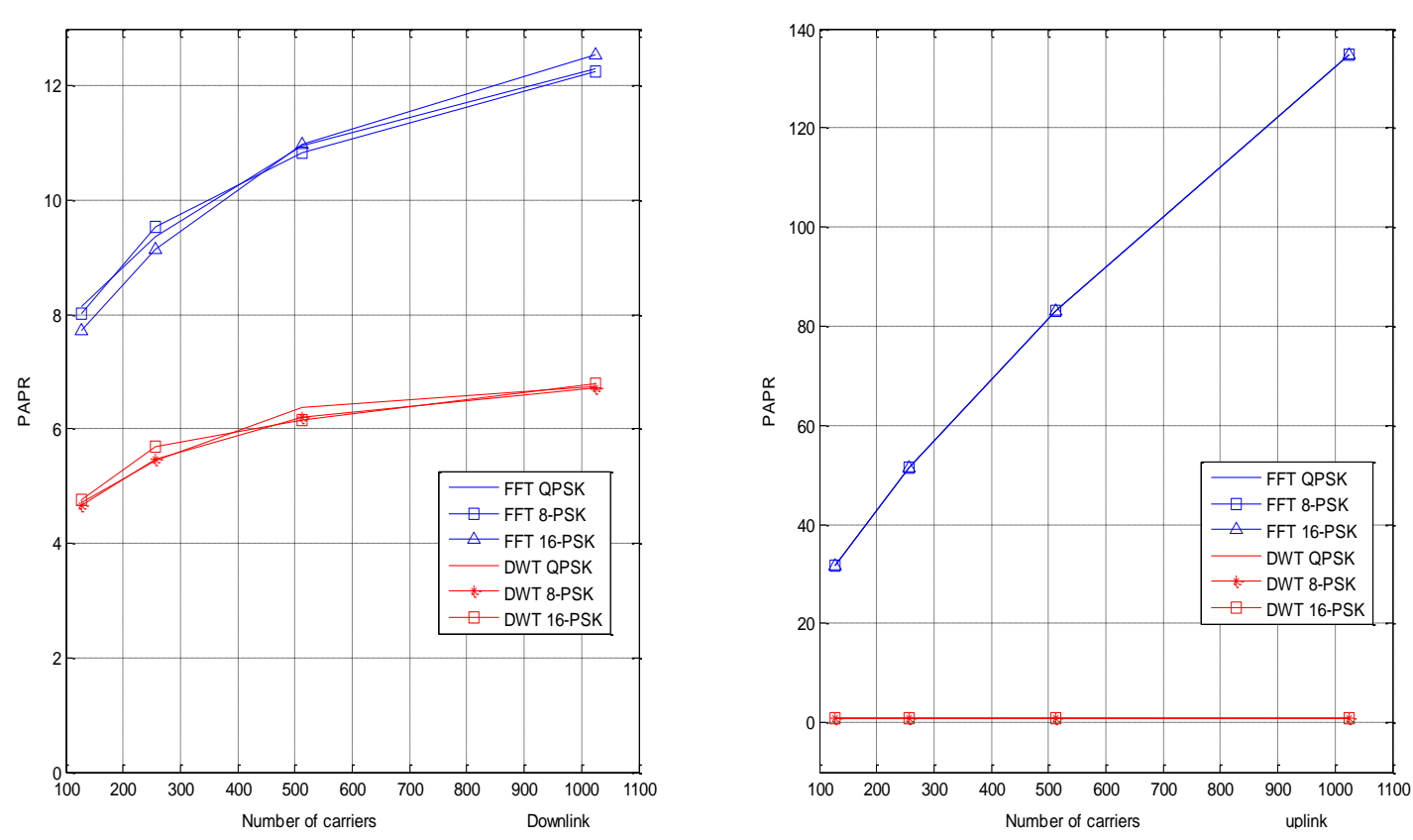

Figure 7. PAPR performance in downlink and uplink transmission with different number of carriers

\section{CONCLUSION}

In this paper, a full comparison between the performance of the wavelet based MC-CDMA system and the Fourier based MC-CDMA system over the AWGN in terms of BER and PAPR was made. The results shows that the Wavelet based system performs a way better than the Fourier based one in terms of $B E R$ for different modulation techniques and different number of sub carriers. Moreover, the $P A P R$ of the wavelet system is noticeably lower and invariant with number of carriers compared with Fourier one in both the downlink and uplink transmission modes. Accordingly, the wavelet based MC-CDMA is strongly expected to replace the Fourier based system in both research and implementation fields.

The research could be expanded to measure the performance of the full user capacity of the two systems in carrier modulated case, study the effect of Doppler shift and consider other types of more complex channels such as Rayleigh Fading channel. Moreover, the traditional Fourier based MC-CDMA system could be compared with other wavelet based MC-CDMA system that uses Wavelet Packet Transform WPT instead of DWT in which both the detail and approximation coefficients are decomposed to create the full binary tree.

\section{REFERENCES}

[1] D. Tse and P. Viswanath, "Fundamentals of Wireless Communications," Cambridge university press, New York, 2005.

[2] K. Fazel and S. Kaiser, "Multicarrier and Spread Spectrum System from OFDM and MC-CDMA to LTE and WiMAX," John Wiley \& Sons L.td, UK, 2008.

[3] S. C. Yang, "OFDMA System Analysis and Deign," Artech House, USA, 2010.

[4] N. Hamad, "OFDMA Performance Measurements in WiMAX Physical Layer Network," International Journal of Applied Engineering Research, vol/issue: 10(24), pp. 44393-44398, 2015.

[5] G. Gowri, et al., "Performance Analysis of DWT-OFDM and FFT-OFDM Systems," International Journal of Engineering and Technology, vol/issue: 5(2), pp. 1455-1461, 2013. 
[6] N. Hamad, "A New Proposal of Handover Algorithm between Cellular Mobile and Mobile WiMAX Systems," International Journal of Applied Engineering Research, vol/issue: 11(24), pp. 11894-11900, 2016.

[7] Y. Li and G. L. Stiiber, "Orthogonal Frequency Division Multiplexing for Wirele Communications," Springer, USA, 2006.

[8] M. Gerami, “A Survey on WiMax," International Journal of Computer Science and Information Security (IJCSIS), vol/issue: 8(2), pp. 352-357, 2010.

[9] S. Tripathi, et al., "PAPR Reduction in OFDM System using DWT with Non-linear High Power Amplifier," International Journal of Innovative Technology and Exploring Engineering, vol/issue: 2(5), pp. 184-188, 2013.

[10] R. Mirghani and M. Ghavami, "Comparison between Wavelet-based and Fourier-based Multicarrier UWB Systems," Institute of Eng. and Tech. Comm., vol/issue: 2(2), pp. 353-358, 2008.

[11] S. Jajula and P. V. Ramana, "Effects of Nonlinearity on DFT-OFDM and DWT-OFDM Systems," International Journal of Eng. Trends and Technology, vol/issue: 4(6), pp. 2603- 2607, 2013.

[12] A. Kiani and S. Mousavi, "Performance Assessment of DFT-OFDM and DWT-OFDM Systems in the Presence of the SSPA and Fading Channel," Inter. Journal of Computer Networks and Comm. Security, vol/issue: 1(7), pp. 316-323, 2013.

[13] R. Bodhe, et al., "Performance Comparison of FFT and DWT based OFDM and Selection of Mother Wavelet for OFDM," Inter. Journal of Computer Science and IT, vol/issue: 3(3), pp. 3993-3997, 2012.

[14] K. Trivedi, et al., "BER Performance of OFDM With Discrete Wavelet Transform for Time Dispersive Channel," IJRET: International Journal of Research in Engineering and Technology, vol/issue: 3(10), pp. 152-155, 2014.

[15] J. Givary, et al., "Analysis Effect of Discrete Wavelet Transform in Multi Carrier Code Division Multiple Access," The 2016 IEEE Asia Pacific Conference on Wireless and Mobile (APWiMob), pp. 99-103, 2016.

[16] P. Manhas and M. K. Soni, "OFDM PAPR Reduction Using Recurring SLM with Cyclic and Linear Block Codes Schemes," TELKOMNIKA Indonesian Journal of Electrical Engineering, vol/issue: 16(1), pp. 1-6, 2015.

[17] F. H. Juwono, et al., "A Study on Peak-to-Average Power Ratio in DWT-OFDM Systems," TELKOMNIKA Indonesian Journal of Electrical Engineering, vol/issue: 12(5), pp. 3955-3961, 2014.

[18] S. G. Mallat, "Mutiresolution Approximations and Wavelet Orthonormal Baes of $L^{2}(R)$," Ttransactions of the American Mathematical Society, vol/issue: 315(1), pp. 69-87, 1989.

[19] S. Verdu, "Multiuser Detection," Cambridge University Press, UK., 1998.

[20] H. Nasser and T. Hashimoto, "Reverse link capacity of a wireless multimedia CDMA system with power and processing gain control in conjunction with CCI canceller," IEICE Trans. Fundamentals, vol/issue: 84(A), pp. 347-355, 2001.

\section{BIOGRAPHIES OF AUTHORS}

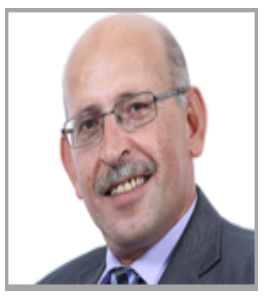

Nasser A. Hamad was born Nablus, Palestine on August 15, 1967. He received the MSC and $\mathrm{PhD}$. from the University of Electro-Communications, Tokyo, Japan, respectively, in 1998, and 2001. He is now an Associate professor in the American University of Ras Al Khaimah, Ras Al Khaimah, UAE. His main interests are on information theory and its applications. Cellular mobile systems and data security and steganography techniques.

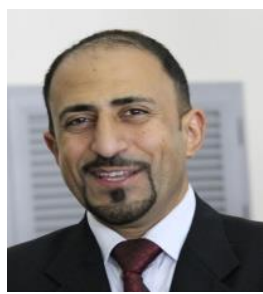

Maen Takruri received his $\mathrm{PhD}$ in Electrical Engineering from University of Technology (UTS), Sydney in 2010. He also received his BSc and MSc degrees in Electrical Engineering from the University of Jordan. Currently, he is an Associate Professor and the Chairman of the Department of Electrical, Electronics and Communications Engineering at the American University of Ras Al Khaimah (AURAK). He has a wide spectrum of research interests that include signal processing and data fusion, estimation theory and target tracking, biomedical systems, machine learning and image processing.

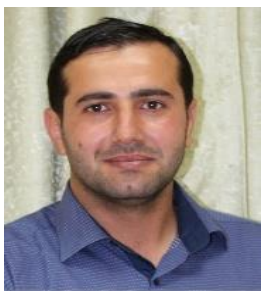

Mahdi Barhoush receivd his MSc in Electrical Engineering, Informaion Technology and Computer Engineering from RWTH Aachen - Germany in 2015. He received his BSc in Telecommunication Engineering from the Arab American University - Palestine. His main intrests include Cyper-Physical systems, Information Theory, Distributed Optimization and Networking. 